\title{
Evaluation of a Hydrophobic-Hydrophilic (Hydrogenated Castor Oil-Gum Tragacanth) Matrix using Propranolol Hydrochloride as Test Drug
}

\author{
R. T. J. CHIGWANDA* AND P. R. CHINOPFUKUTWA
}

School of Pharmacy, University of Zimbabwe, P.O. Box MP 167, Mt Pleasant, Harare, Zimbabwe.

\begin{abstract}
An evaluation of propranolol hydrochloride release from hydrogenated castor oilgum tragacanth matrices was carried out. The $\mathrm{pH}$ of the dissolution medium was found to affect propranolol hydrochloride release from such matrices. Acidic $\mathrm{pH}$ was found to enhance release while alkaline $\mathrm{pH}$ retarded release. This was attributed to possibly the effect of gum tragacanth matrix material or the propranolol hydrochloride itself. Either square root of time kinetics or first order kinetics could explain propranolol hydrochloride release mechanism from the hydrogenated castor oil-gum tragacanth matrices. Addition of hydrophobic hydrogenated castor oil matrix material to the hydrophilic gum tragacanth matrix was found to enhance release. This was attributed to possibly the disruption of the gum tragacanth matrix by the hydrophobic hydrogenated castor oil matrix material. Although gum tragacanth is primarily a hydrophilic material, it was found to have better release sustaining characteristics than the hydrophobic matrix material hydrogenated castor oil.
\end{abstract}

Key words: Hydrogenated castor oil, gum tragacanth, matrix, hydrophobic, hydrophilic

\section{INTRODUCTION}

Hydrogenated vegetable oil matrices such as those made from hydrogenated castor oil (HCO) release drug primarily according to square root of time kinetics:

$$
\mathrm{Q}=\mathrm{kt}^{0.5}
$$

Where $Q$ is the cumulative amount of drug released at time $t$ and $k$ is the square root of time kinetics rate constant [1].

Such devices remain intact post releasing the active ingredient and hence are termed monolithic systems [2]. Hydrogenated castor oil can also be termed a hydrophobic matrix material [2].

On the other hand, gum tragacanth (GT) can be termed a natural occurring hydrophilic matrix material [3]. Drug release from such matrices has been found to follow either first order kinetics:

$$
\log _{10}(1-Q)=k t
$$

Where $Q$ is the cumulative fraction of drug released at time $t$ and $k$ is the first order rate constant or square root of time kinetics [3].
While HCO matrices form monolithic systems, GT matrices eventually erode with time.

The present study was aimed at evaluating release characteristics of a combination matrix of $\mathrm{HCO}$ and GT using propranolol hydrochloride as a test drug.

\section{METHODS AND MATERIALS}

\section{Materials}

Propranolol hydrochloride was provided by Varichem Pharmaceutical Company (Harare, Zimbabwe) while $\mathrm{HCO}$ was supplied by Croda Chemical Company (Harare, Zimbabwe). Gum tragacanth was obtained from Sigma Chemical Company (St Louis, MO, USA).

\section{Preparation of the tablets}

Tablets were prepared by direct compression. The HCO, GT and propranolol hydrochloride were mixed in the appropriate ratios and directly compressed on a lubricated Erweka single punch tablet machine (Type EK 0 , $\mathrm{GmbH}$, Heusenstamm, Germany). Each tablet mixture 
was individually weighed prior to compression so that tablets of approximately $200 \mathrm{mg}$ are obtained. All tablets were made at a compaction force of 7.5 units. The drug content was $30 \%$ w/w.

\section{Dissolution studies}

Dissolution studies were carried out according to the BP Apparatus I (Basket Apparatus) method [4]. For each run, 3 tablets were analyzed. Drug content was analyzed using an ultravioletvisible spectrophotometer (Shimadzu, UV 1601, Kyoto, Japan).

\section{Treatment of data}

Release profiles were plotted for the data obtained. The release data was also analyzed according to zero order, first order and square root of time kinetics. The correlation coefficients obtained were subjected to the Student's t-test and the results analyzed at the $5 \%$ level.

\section{RESULTS AND DISCUSSION}

The summary of the release kinetics of propranolol hydrochloride from the various matrices is shown in Table 1. Release from GT and HCO:GT matrices could be explained by either square root of time kinetics or first order kinetics as the correlation coefficients for these two mechanisms were not significantly different at the $5 \%$ level as shown in Table 1 . However, propranolol hydrochloride release from $\mathrm{HCO}$ matrices was best explained by first order kinetics as the two correlation coefficients were significantly different at the $5 \%$ level. Zero order kinetics had no significant role in the drug release mechanism of these matrices. This was to be expected since a previous study had shown that GT matrices release drug via either square root of time or first order kinetics [3]. However, in the present study, propranolol hydrochloride release from $\mathrm{HCO}$ matrices rather than follow square root of time kinetics as shown earlier [1], followed first order kinetics. Usually monolithic drug delivery devices follow square root of time kinetics because the distance that the drug in the inner layers has to travel increases with time and hence a reduction in release rate with time. In the case of first order kinetics, the drug release is dependent on the amount of drug remaining in the device at each corresponding time.

Propranolol hydrochloride was released better in acidic $\mathrm{pH}$ than in alkaline $\mathrm{pH}$ from the HCO:GT (1:1) matrices as shown in Figure 1. The pKa of propranolol hydrochloride is approximately 9.5 . According to the Henderson-Hasselbach equation, at $\mathrm{pH} 2.5$, propranolol hydrochloride is almost $100 \%$ ionized while at $\mathrm{pH} 7.6$ it is $98.76 \%$ ionized. The $\mathrm{pH}$ of distilled water is approximately 6.5 hence intermediate releases as shown in Figure 1. This difference in percentage ionization with $\mathrm{pH}$ might explain the difference in the release profiles. However, previous studies have shown that drug release (including propranolol hydrochloride) from $\mathrm{HCO}$ matrix devices is not affected by $\mathrm{pH}[5,6]$. Therefore it is possible that this $\mathrm{pH}$ effect on release is due to the GT component of the matrices rather than the drug propranolol hydrochloride.

Figure 2 depicts the effect of the type of matrix on propranolol hydrochloride release. As the content of HCO increased, propranolol hydrochloride release increased as well. This implies that GT had better release sustaining characteristics than $\mathrm{HCO}$ although it is a hydrophilic matrix material. The $\mathrm{HCO}$ forms monolithic drug delivery systems and drug release occurs primarily by diffusion through tortuous pores [1]. On the other hand, release of drugs from hydrophilic matrices such as GT may be controlled by diffusion, slow dissolution, erosion of the matrix or by a combination of processes [7-9]. On contact with the dissolution medium, the majority of hydrophilic devices start swelling and the tablet thickness increases. Soon thereafter, the polymer and drug dissolution start to occur. If the drug diffusion and polymer erosion are synchronized, zero order release kinetics may be obtained. Due to polymer dissolution or dispersion, the distance that the drug travels before elution might remain constant resulting in constant release rates. However, in practice this synchronization rarely occurs hence drug release from hydrophilic matrices usually occurs via either square root of time kinetics or first order kinetics as obtained in the present study. Nevertheless, adding a 
hydrophobic matrix material such as $\mathrm{HCO}$ to GT matrix enhances drug release. This is probably because HCO disrupts the GT matrix and facilitates drug release.

Table 1: Correlation coefficient values for square root and first order release kinetics for the various matrices and the Student's t-test results

\begin{tabular}{ccccc}
\hline $\begin{array}{c}\text { Matrix } \\
\text { Type }\end{array}$ & $\begin{array}{c}\text { Correlation coefficient } \\
\text { Square root of time } \\
\text { kinetics }\end{array}$ & $\begin{array}{c}\text { First order } \\
\text { kinetics }\end{array}$ & & Student's t-test results \\
\cline { 2 - 4 } HCO & $0.96211 \pm 0.00537$ & $0.98393 \pm 0.00360$ & $\begin{array}{c}\mathrm{T}=5.8457 \\
\mathrm{p}=0.0043\end{array}$ & $\begin{array}{c}\text { Significantly different at } \\
0.05 \text { level }\end{array}$ \\
GT & $0.99733 \pm 0.00110$ & $0.99723 \pm 0.00116$ & $\begin{array}{c}\mathrm{T}=0.1049 \\
\mathrm{p}=0.9215\end{array}$ & $\begin{array}{c}\text { Not significantly } \\
\text { different at } 0.05 \text { level }\end{array}$ \\
$\begin{array}{c}\text { HCO:GT } \\
(8: 2)\end{array}$ & $0.87947 \pm 0.00795$ & $0.89616 \pm 0.01478$ & $\begin{array}{c}\mathrm{T}=1.7224 \\
\mathrm{p}=0.1601\end{array}$ & $\begin{array}{c}\text { Not significantly } \\
\text { different at } 0.05 \text { level }\end{array}$ \\
$\begin{array}{c}\text { HCO:GT } \\
(7: 3)\end{array}$ & $0.98636 \pm 0.00411$ & $0.99100 \pm 0.00222$ & $\mathrm{~T}=1.7283$ & $\begin{array}{c}\text { Not significantly } \\
\mathrm{p}=\mathbf{0 . 1 5 9 0}\end{array}$ \\
$\begin{array}{c}\text { HCO:GT } \\
(6: 4)\end{array}$ & $0.99688 \pm 0.00068$ & $0.99866 \pm 0.00115$ & $\begin{array}{c}\mathrm{T}=2.3049 \\
\mathrm{p}=0.0825\end{array}$ & $\begin{array}{c}\text { Not significantly } \\
\text { different at } 0.05 \text { level }\end{array}$ \\
$\begin{array}{c}\text { HCO:GT } \\
(1: 1)\end{array}$ & $0.99438 \pm 0.00326$ & $0.99399 \pm 0.00245$ & $\begin{array}{c}\mathrm{T}=0.16701 \\
\mathrm{p}=0.8755\end{array}$ & $\begin{array}{c}\text { Not significantly } \\
\text { different at } 0.05 \text { level }\end{array}$ \\
\hline
\end{tabular}

Where $T$ is the Student's t-test and $p$ is the probability value.

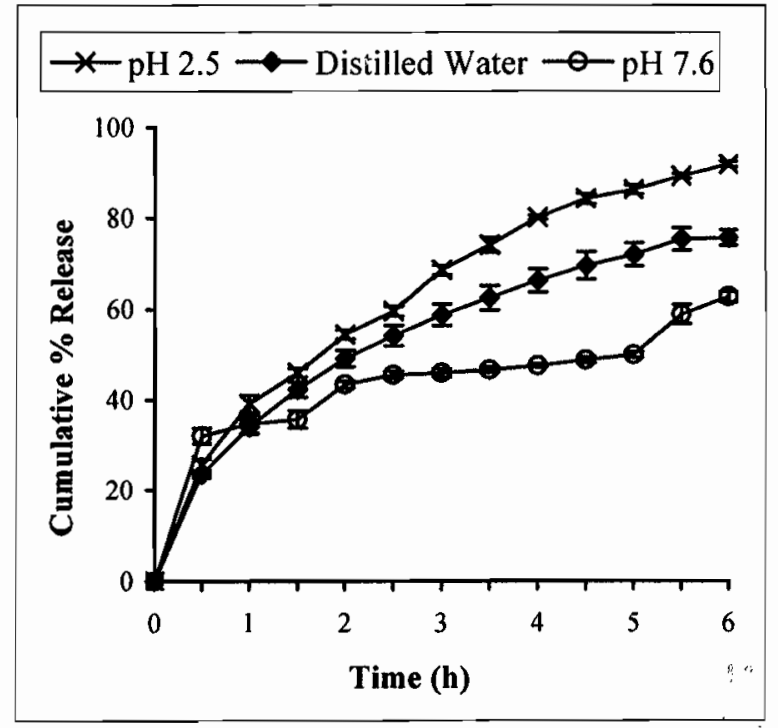

Figure 1: Effect of $\mathrm{pH}$ on Propranolol $\mathrm{HCl}$ release from matrices made from Hydrogenated Castor Oil: Gum Tragacanth (1:1)

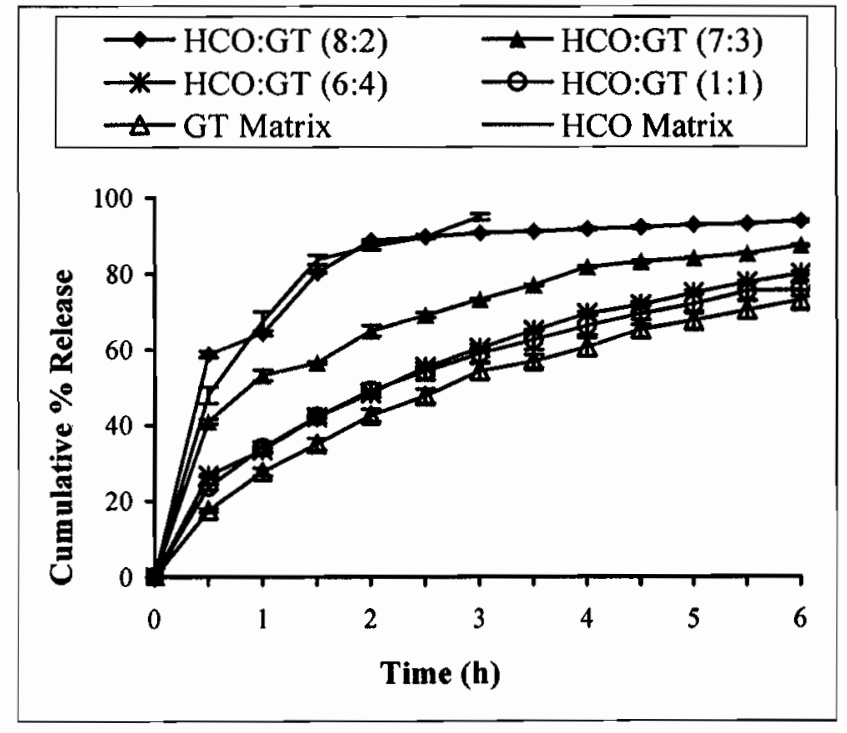

Figure 2 : Effect of type of matrix on Propranolol $\mathrm{HCl}$ release. 


\section{ACKNOWLEDGEMENTS}

The authors are grateful to the Research Board, University of Zimbabwe, for sponsoring part of the research.

\section{REFERENCES}

[1] R.T.J. Chigwanda and J. N. Staniforth, East Cent. Afr. J. Pharm. Sci. 3 (2000) 48-51.

[2] R.T.J. Chigwanda and J.N. Staniforth, East Cent. Afr. J. Pharm. Sci. 3 (2000) 61-65.

[3] R.T.J. Chigwanda and I. Pfaira, East Cent. Afr. J. Pharm. Sci. 2 (1999) 16-19.
[4] British Pharmacopoeia, 2001.

[5] R.T.J. Chigwanda, PhD Thesis, University of Bath, England, 1995.

[6] P. Lockwood, PhD Thesis, University of Bath, England, 1993.

[7] P. Khullar, R.K. Khar and S.P. Agarwal, Drug Development \& Industrial Pharmacy, 24 (1998) 1095-1099.

[8] C.J. Kim, J. Pharm. Sci. 84 (1995) 303306.

[9] M. Otsuka and Y. Matsuda, J. Pharm. Sci. 84 (1995) 443-447. 